\title{
HIV-exposed, uninfected infants in Uganda experience poorer growth and body composition trajectories than HIV-unexposed infants
}

\author{
Charlotte E. Lane, BA ${ }^{1}$, Elizabeth M. Widen, $\mathrm{PhD}^{2}$, Shalean M. Collins, MPH, RD ${ }^{3}$, Sera L. \\ Young, $\mathrm{PhD}^{3,4}$ \\ 1. University of North Carolina at Chapel Hill, Department of Nutrition \\ 2.University of Texas at Austin, Department of Nutritional Science \\ 3.Northwestern University, Department of Anthropology \\ 4. Northwestern University, Institute for Policy Research
}

\section{Abstract}

Background: HIV-uninfected infants of HIV-positive women may experience worse growth and health outcomes than infants of HIV-negative women, but this has not been thoroughly investigated under the WHO's most recent recommendations to reduce vertical transmission.

Objective: To determine if HIV-exposed and -uninfected (HEU) infants whose mothers received Option B+ have higher odds of experiencing suboptimal growth trajectories than HIV-unexposed, uninfected infants and if this relationship is affected by food insecurity.

Design: Repeated anthropometric measures were taken on 238 infants (HEU=86) at 1 week and $1,3,6,9$, and 12 months after delivery in Gulu, Uganda. Latent class growth mixture modeling was used to develop trajectories for length-for-age z-scores (LAZ), weight-for-length z-scores, midupper arm circumference (MUAC), sum of skinfolds, and arm fat area. Multinomial logistic models were built to predict odds of trajectory class membership, controlling for socioeconomic factors.

Results: HEU infants had greater odds of being in the shortest two LAZ trajectory classes $(\mathrm{OR}=3.80[1.22,11.82], \mathrm{OR}=8.72[1.80,42.09])$ and higher odds of being in smallest sum of skinfolds trajectory class $(\mathrm{OR}=3.85[1.39,10.59])$ vs. unexposed infants. Among HEU infants, increasing food insecurity was associated with lower odds of being in the lowest sum of skinfolds class $(\mathrm{OR}=0.86[0.76,0.98])$.

Conclusions: There continues to be differences in growth patterns by HIV-exposure under the new set of WHO guidelines for the prevention of mother-to-child transmission of HIV and the feeding of HEU infants in low-resource settings that are not readily identified through traditional

Corresponding Author: Sera L. Young, 1819 Hinman Avenue, 1(847)467-2174, Evanston, Illinois 60208.

Conflict of interest statement: The authors report no conflicts of interest

Clinical trial registry: clinicaltrials.gov NCT02922829 and NCT02925429 
mixed effects modeling. Food insecurity was not associated with class membership, but differentially affected adiposity by HIV-exposure status.

\section{Keywords}

HIV-exposed and -uninfected; anthropometry; HAZ; LAZ; sum of skinfolds; MUAC

\section{Introduction}

HIV-exposed, uninfected (HEU) infants may be at increased risk of a number of adverse outcomes. Although their health has been shown to be markedly better than those living with HIV, there is evidence to suggest that, before widespread antiretroviral therapy (ART), HEU infants experienced worse growth and increased risk of morbidity and mortality compared to their HIV-unexposed counterparts. ${ }^{1-9}$

HEU infants may also be at increased risk of experiencing the negative consequences of food insecurity. This is both because HIV is often associated with increased food insecurity, 10 and food insecurity is associated with myriad adverse outcomes. ${ }^{11,12}$ These include worse maternal body composition, exclusivity of breastfeeding, perceived breast milk quality and quantity, and maternal stress. ${ }^{13-15}$

Over the last decade, the World Health Organization (WHO) has made several changes to its recommendations for the prevention of mother-to-child transmission (MTCT) of HIV and the feeding of HEU infants in low-resource settings. Since 2012, the WHO recommends Option B+: universal, lifelong, antiretroviral therapy (ART) for all HIV-positive pregnant or breastfeeding women. ${ }^{16}$ It further recommends that HEU infants in low-resource settings be exclusively breastfed for six months and that breastfeeding continues for at least one year. ${ }^{17}$ The effects of intrauterine exposure to HIV and ART on infant birth outcomes and subsequent growth remain unknown; however, studies indicate potentially harmful impacts of the virus and/or medicines. ${ }^{1,18-22}$

The reasons for the historically worse growth of HEU infants are not known. Some have posited environmental exposures after delivery, ${ }^{1,2,7,9,23-25}$ others suggest molecular metabolic abnormalities since conception, ${ }^{18,26,27}$ or even alterations in the microbiome. ${ }^{28}$ However, because the current recommendations have only been recently adopted, it is unclear whether Option B+ or recommendations for prolonged breastfeeding have been successful in eliminating the differences between HEU and HIV-unexposed and -uninfected (HUU) infants in health and wellbeing.

To our knowledge, three studies to date have considered the growth trajectories of HEU infants under the new guidelines. They suggest that growth differences persist, but conclusions are limited given their divergent methodologies. For example, HEU infants in Kigali, Rwanda participating in a study of Option B+ and breastfeeding for up to two years had low length-for-age and slightly elevated weight-for-length. ${ }^{29}$ However, this study did not include HUU comparator infants; comparisons were relative to the WHO standard throughout the first two years of life. Two studies have used mixed effects modeling to contrast the growth patterns of HEU infants treated under current WHO recommendations to 
comparable HUU infants. One study showed that HEU infants in urban Nigeria were at higher risk of stunting and underweight than comparable HUU infants. ${ }^{30}$ The second, in South Africa, found that HEU infants had slower linear growth than their HIV-unexposed counterparts. ${ }^{31}$ However, body composition measures such as skinfold assessments, midupper arm circumference (MUAC), or arm fat area (AFA) were not taken in either study, and analyses were limited to longitudinal, multilevel regression modeling. Regression-based longitudinal approaches assume that there is a single, typical growth pattern for the entire population. However, if the underlying biological mechanisms and risk factors act differently within subgroups of a population, for example within HIV-exposed and unexposed infants, the assumption of a single underlying distribution can be problematic. 32,33

Therefore, in order to better understand differences in growth patterns between HEU and HUU infants, we first compared length-for-age z-scores (LAZ), weight-for-length z-scores (WLZ), MUAC, sum of skinfolds, and AFA among otherwise comparable HEU and HUU infants in northern Uganda. In order to examine heterogeneity in growth patterns, we used latent class growth mixture modeling to identify infants' growth trajectories. Latent class growth mixture modeling (sometimes shortened to 'trajectory modeling') is a statistical approach to classifying outcomes based on their patterns across time. Trajectory modeling is more flexible than traditional longitudinal modeling because it allows for the identification of subgroups whose curves can take on different shapes, rather than being forced to remain parallel, as in traditional regression analyses. ${ }^{33}$ Here, we use trajectory modeling to group infants with similar growth patterns into what are called 'classes'. With the creation of classes, the mean growth patterns of each class can be graphically represented and predictors of class membership can be determined using regression techniques.

We hypothesized that, relative to HUU infants, HEU infants would be at increased risk of belonging to classes that experienced poor growth trajectories for LAZ, WLZ, MUAC, sum of skinfolds, and arm fat area (AFA). We further hypothesized that greater food insecurity would be related to increased risk of belonging to classes that experienced poor growth.

\section{Methods}

The Postnatal Nutrition and Psychosocial Health Outcomes Study (PostNAPS) took place at the Gulu Regional Referral Hospital (GRRH) in Gulu, Uganda from October 2012 to January 2015. PostNAPS is the follow-on to PreNAPS, a longitudinal study that examined the relationships between food insecurity, psychosocial health, and nutritional status during pregnancy and postpartum (clinicaltrials.gov NCT02922829 and NCT02925429).

Details on study procedures and ART are described elsewhere. ${ }^{34-37}$ Briefly, women $(n=403)$ were invited to enroll in the Prenatal Nutrition and Psychosocial Health Outcomes Study (PreNAPS) if they were seeking care at GRRH, had a gestational age between 10 and 26 weeks (assessed using last menstrual period), lived within 30 kilometers of the hospital, and knew their HIV status. HIV-infected women were oversampled from the general population with a ratio of 2 to 1 (HIV-uninfected: HIV-infected), such that the prevalence of HIV among women in this study is higher than the general population. In accordance with 
Ugandan national policy, all women at the GRRH received free antenatal care and medications. Additionally, upon presenting at the antenatal care clinic, HIV-positive women received ART and sulfamethoxazole trimethoprim (Septrin: Aspen Pharmacare).

At the PreNAPS enrollment visit, demographic data including last menstrual period, education, marital status, urban/rural residency, age, and previous displacement (due to the 20-year protracted civil war), were collected. A household asset index was derived using principal components analysis from the self-report of household assets based on the Ugandan National Panel Survey 2009/2010, ${ }^{14}$ with higher scores indicating greater wealth. Food insecurity data were collected using the 9-item Individual Food Insecurity Access Scale ${ }^{36}$ during all prenatal and postnatal visits (range: 0-27); higher scores indicate greater food insecurity. We used the food insecurity score from the last prenatal visit as it is most likely to reflect food security status throughout the study period. Exclusive breastfeeding at one week, three months, and six months was determined through a series of questions about breastfeeding and receipt of non-human milk foods since the last visit, and scored as binary (yes/no).

Visits were conducted at one week and 1,3,6,9, and 12 months postpartum. Maternal height, weight, MUAC, and waist circumference were taken at all postpartum visits. Infant weight, length, MUAC, and head circumference were taken at enrollment and all subsequent visits. Infant skinfold data collection for all infants began at one month postpartum. Trained research staff collected anthropometric measures in duplicate; if there was substantial difference in the first two measurements, a third measure was collected. Weight was collected using a digital scale (Seca 874; Seca North America), height/length using a wallmounted stadiometer and recumbent boards (Seca 206/Seca 417; Seca North America), and mid-upper arm circumference (MUAC) using a non-stretchable, retractable tape measure. Subscapular, triceps, suprailiac, and mid-thigh skinfold thickness measures were obtained on the right side of the body using Harpenden calipers (Baty International).

All 246 PreNAPS participants who delivered singleton births after 9 May 2013 were eligible and agreed to participate in PostNAPS (Supplemental Figure 1). Birthweight data were collected at enrollment, and infant body composition assessments were collected at enrollment in July 2013; repeat skinfold and AFA measures were available for 234 infants. Eight infants were dropped because they had less than two visits or were missing baseline data, resulting in an analytic sample of 238 infants (86 HEU and $152 \mathrm{HUU}$ ).

At baseline, $51 \mathrm{HIV}$-infected women had not received HIV medicines and initiated ART with a regimen of tenofovir, lamivudine, and evfavirenz (TDF/3TC/EFV). Of the 35 women who were diagnosed prior to the index pregnancy, 11 reported taking AZT, 26 received NVP, 2 reported 3TC, 1 reported HAART. One woman reported receiving a second-line treatment. Infants received $480 \mathrm{mg}$ Septrin daily until HIV results were confirmed to be negative; HEU infants also received Nevirapine daily for the first 6 months of life.

The Institutional Review Boards (IRBs) at Cornell University and Gulu University approved the study procedures for PreNAPS. These two IRBs and the IRB at Weill Cornell Medical College approved PostNAPS procedures. Permission to carry out the study in Uganda was 
granted by the Ugandan National Council for Science and Technology (UNCST). All mothers provided written informed consent for both PreNAPS and PostNAPS.

\section{Statistical analysis}

LAZ and WLZ were calculated using the WHO igrowup macro in $\mathrm{R}^{38}$ Basic descriptives were then calculated and $t$-tests were used to compare values between HIV-exposed and unexposed infants.

Latent trajectories were developed for LAZ, WLZ, MUAC, sum of skinfolds, and AFA using the lcmm package in $\mathrm{R} .{ }^{39}$ Trajectory modeling groups individuals by the development of a certain outcome over time. ${ }^{33}$ In contrast to traditional approaches to growth modeling, which assume that all infants come from the same underlying distribution and that growth curves are simply shifted from one another, this approach allows for growth curves of subpopulations or 'classes' to take on fundamentally different shapes. Therefore, with this approach, we can investigate not only if HEU infants and HUU infants experience different growth at particular time points, but if their growth curves have different shapes. Trajectory models require only specification of the expected relationship between the outcome and time. ${ }^{33}$ Three types of time relationships (in this case, infant age) were considered in the development of growth trajectories: age and age ${ }^{2}$ terms with a linear link, age and age ${ }^{2}$ terms with a spline link, and age with a spline link (with four equidistant knots). Models specifying two to seven classes were considered. Due to limited variation at younger ages, a $\log$ transformation was applied to MUAC and arm fat area values.

We only considered models in which no class contained less than $9 \%$ of the population because we wanted classes to actually represent groups of infants rather than isolate one or two infants as different. Among these models, we chose those with the best fit as measured by BIC (Supplemental Table 1). For all measures, the age and age ${ }^{2}$ spline models had the best fit. Infants were assigned to the latent class to which they had the highest probability of belonging for each growth parameter.

Mean growth trajectories for each class were then plotted in R using the ggplot2 package. ${ }^{40}$ To understand how these growth parameters were interrelated, the relationship between class membership in any two of the anthropometric measures (LAZ, WLZ, MUAC, sum of skinfolds, and AFA) was examined using ANOVA.

Multinomial logistic regressions were used to consider the relationship between odds of growth trajectory class membership and HIV status and food insecurity, as well as maternal education, height, age, employment, previous maternal displacement, infant sex, season of birth, asset index, gestational age, and exclusive breastfeeding to three months. ${ }^{41}$ The breastfeeding status of six infants was unknown at three months and they were dropped from analytic sample for multinomial logistic regression; no other values for covariates were missing. An interaction term was included between food security and HIV status to allow for the relationship between food security and odds of class membership to vary by HIV exposure status. The class that contained the most infants was used as the referent in order to increase power. 
Finally, in order to compare the trajectory modeling approach with traditional regression, we used the lme4 package in $\mathrm{R}$ to conduct a mixed effects longitudinal analysis in a post hoc analysis. ${ }^{42}$ We examined the relationships between the same set of independent variables and LAZ and sum of skinfolds only, because these were the two growth trajectories for which HIV-exposure was statistically significant.

\section{Results}

A total of 238 infants had baseline data and at least two repeated, simultaneous length and weight measures (Supplemental Figure 1). The mean number of study visits was 5.77. Mothers of HEU infants reported lower asset and higher food insecurity scores (Table 1). Food insecurity scores were generally consistent over the study period, with mean values ranging from a high of 6.47 at the first postnatal visit to a low of 5.43 at the last visit. There was no statistically significant difference in the mean values of the food insecurity scores reported at the last prenatal visit or any of the subsequent visits (ANOVA $\mathrm{p}=0.212$ ). HEU infants were more likely to be exclusively breastfed at 1 week and 3 months postpartum, but this difference was no longer significant at 6 months.

In cross-sectional descriptive statistics at one month of age, HEU infants were somewhat shorter and thinner than HUU infants (Table 1). HEU infants had mean LAZ that were -0.5 units shorter for their age and sex than unexposed infants, and had sum of skinfold measures that averaged 1.2 centimeters smaller. HEU infants were significantly shorter over the whole period (Figure 1).

Four latent growth trajectories in LAZ and WLZ, and three latent growth trajectories emerged for MUAC, sum of skinfolds, and AFA (Figure 2). Sum of skinfold and AFA-class membership were significantly associated with LAZ and WLZ-class membership (Supplemental Table 2). For example, infants in the middle classes for sum of skinfolds and AFA tended to be in the tall-mid class for LAZ, and tended to belong to the middle WLZclass. Infants in the low AFA class tended to be in the short-mid LAZ-class and the low or rapidly falling WLZ-classes.

HEU infants had higher odds of belonging to LAZ-classes that experienced suboptimal growth than HUU infants (Figure 2, Table 2). In the final multivariate models, HEU infants had higher odds than HUU infants of being in either of the two shortest trajectory classes than the second tallest class, the referent (Table 2).

Among the 234 infants with repeated skinfold measures, HEU infants also tended to have worse patterns in the growth of their sum of skinfold measures than HUU infants (Figure 2). HEU infants had increased odds of being in the thinnest class of sum of skinfolds; $44 \%$ of infants in the lowest sum of skinfolds class were HEU (Table 3). Although latent growth trajectories were developed for WLZ, MUAC, and AFA there was no difference in odds of class membership by exposure status (Supplemental Tables 3,4,5).

In order to see how results differed by analytic technique, we used mixed effects regression to analyze the two growth parameters which significantly differed by HIV-exposure status, LAZ and sum of skinfolds. In this analysis, HEU infants were shorter than HUU infants 
(consistent with what we found in trajectory models), but there was no longer a difference in sum of skinfolds.

There were no significant relationships between food insecurity and trajectory membership. However, among HEU infants, greater food insecurity was associated with lower odds of being in the lowest sum of skinfolds class (OR interaction $=0.86[0.76,0.98])($ Table 3$)$.

\section{Discussion}

In this cohort of HUU and HEU Ugandan infants whose mothers received Option B+, trajectory modeling revealed important differences in outcomes for LAZ and sum of skinfolds that would have been otherwise been obscured using basic descriptive statistics or traditional regression models.

In simple cross-sectional comparisons between HEU and HUU at one month, HEU infants had lower LAZ and smaller sum of skinfolds than HUU infants, and these differences dissipated by 9.5 months (Table 1 ).

However, using latent class growth mixture modeling, HEU infants had higher odds of belonging to the two shortest LAZ-classes (Table 2) as well as belonging to the lowest sum of skinfolds class (Table 3) compared to HUU infants across time. In other words, HEU infants consistently experienced growth challenges in both length and adiposity. When we investigated differences in growth patterns using the more conventional longitudinal, mixed effects regression models, HEU infants also experienced statistically significantly worse growth outcomes in terms of LAZ compared to HUU ( $\beta=-0.62$, Supplemental Table 6). However, differences were not seen for sum of skinfolds. This suggests that there are important differences in the shapes of growth curves that neither cross-sectional analyses nor traditional longitudinal models with their strong set of assumptions can capture.

Not only do HEU infants have a higher tendency to be in low LAZ and sum of skinfolds trajectory classes, but there is an association between sum of skinfolds class and LAZ class, indicating that it is the same infants that tend to be small in both of these measures (pvalue $<0.01$, Supplemental Table 2). While it is possible that this relationship simply reflects that shorter infants are also smaller in their skinfold measurements, the (non-significantly) larger WLZ of HEU infants compared to HUU infants at one month suggests that HEU infants are gaining weight at the expected rate relative to their size. Therefore, their subsequent smaller skinfold measures likely indicate differences in the pattern of adipose deposition. Although it might be tempting to dismiss these differences as not meaningful because z-scores become more similar over time, there is extensive evidence that early growth patterns are associated with long-term health risks, even if subsequent catch-up growth is achieved. ${ }^{43,44}$

These altered growth patterns (shorter infants with less subcutaneous fat) suggest that biological mechanisms to conserve nutrient stores may be occurring in HEU infants either in response to ART or the virus itself. Data are not available to investigate possible hormonal or immunological explanations of these mechanisms, but we encourage their exploration in 
future research by collecting serum data on acute phase proteins and other immunological factors.

Thus, to our first hypothesis, we found differences in growth patterns related to HIVexposure under the new set of WHO guidelines for the prevention of MTCT of HIV and the feeding of HEU infants in low-resource settings. Because we controlled for socioeconomic factors, breastfeeding, and gestational age, we believe the most plausible explanation for the differences in growth is a biological effect of HIV and/or ART. Nonetheless, we are unable to make causal claims. Further, we did not find significant differences in MUAC, WLZ, or AFA. It is possible that this is because the underlying mechanisms that affect sum of skinfolds and LAZ is not acting the same way on these measures. More likely, their higher variation, the rapid fluctuation of some indicators (e.g. WLZ) and our small sample sizes simply prevented the identification of these effects. In contrast, length is a well-established indicator of long-term nutritional status. ${ }^{45}$

To our second hypothesis, that greater food insecurity would be associated with poor growth, we found that maternal food insecurity itself did not predict trajectory membership. However, in regards to sum of skinfolds among HEU infants, we found a significant interaction between food insecurity and HIV exposure, such that greater food insecurity was related to decreased odds of being in the lowest class (Table 3). While this could be interpreted to indicate that food insecurity is protective against being in the lowest class, it likely reflects the significantly increased odds of being in this class already experienced by HEU infants due to their exposure status, i.e. food insecurity simply cannot further increase these odds.

Most of the covariates associated with suboptimal growth were consistent with previous literature. For example, greater maternal height and female sex were related to lower odds of being in the two shortest classes (Table 2). This is consistent with our understanding of drivers of child height and with another study of HEU infants in the era of Option B+ in Rwanda. ${ }^{29}$ Data from the Rwandan and Ugandan DHS also show less severe growth deficits in female infants than males. ${ }^{46,47}$

This study has many strengths, including repeated diverse anthropometric measures among comparable groups of HEU and HUU infants. To our knowledge, this is the most detailed comparison of growth in HEU and HUU infants in the same setting, in terms of duration of follow-up, and types and frequency of anthropometric assessments. However, sample size limitations could result in low power and the failure to identify some true associations, e.g. with WLZ, MUAC or arm fat area. In addition, because this is an observational study, there may be residual confounding due to socioeconomic characteristics, other behavioral differences, or the direct biological effect of HIV and/or ART exposure. However, differences in breastfeeding rates are not likely to have caused these differences in outcomes because breastfeeding is accounted for in the models.

In conclusion, HEU infants in northern Uganda on Option B+ experienced worse growth patterns in LAZ and sum of skinfolds than their unexposed counterparts in the first year of life. Food insecurity was not associated with class membership, but differentially affected 
adiposity by HIV-exposure status. While enormous strides have been made to support the health and wellbeing of HEU infants and their mothers, these data suggest need to focus on the gap between the growth of HEU and HUU infants. More information is needed about the drivers of these differences, including physiological and pharmacologic influences, in order to support the health and wellbeing of the growing population of HEU children in sub-

Saharan Africa.

\section{Supplementary Material}

Refer to Web version on PubMed Central for supplementary material.

\section{Acknowledgments}

We are deeply grateful to the mothers and infants who participated in this study, without whom this research would not have been possible. We thank the GRRH antenatal care clinic for providing space for the research team within the hospital. We thank Sophie Becky Ajok, who is the coordinator for the prevention of mother-to-child transmission of HIV services at the GRRH antenatal clinic, for helping to recruit pregnant women to the study; Angela Arbach for assisting with the coordination of the Pre-NAPS; and Nicole Sirotin for her involvement with the PostNAPS. We also thank the following PreNAPS and PostNAPS Uganda staff for their dedication and hard work on this study: Stella Adoch, Gladys Acayo, Hillary Kilama, Joe Cord, Daniel Onen, and Geoffrey Abwola. We also acknowledge the contributions of Barnabas K. Natamba towards the conception, design and supervision of data collection in the PreNAPs and PostNAPs studies.

Sources of support: CL was supported by the Royster Society of Fellows. Data collection was supported by the Feed the Future Innovation Laboratory for Nutrition, which is funded by the United States Agency for International Development (USAID) and based at Tufts University (USAID OAA-L-10- 00006), and by a seed grant for collaborations between Cornell University and Weill Cornell Medical College faculty. EMW was supported by the Eunice Kennedy Shriver National Institute of Child Health and Human Development (K99/R00 HD086304), the National Institute of Diabetes and Digestive and Kidney Diseases (T32 DK091227 and T32 DK007559), and PepsiCo Global R+D (unrestricted grant to support research in maternal and child health). SLY was supported by the National Institute of Mental Health (K01 MH098902). The content is solely the responsibility of the authors and does not necessarily represent the official views of the National Institute of Mental Health or the National Institutes of Health.

\section{Abbreviations:}
AFA
Arm fat area
ART
Antiretroviral therapy
AZT
Azidothymidine
BIC
Baysian information criterion
GRRH
Gulu Regional Referral Hospital
HEU
HIV-exposed and -uninfected
HUU
HIV-unexposed and -uninfected
IRB
Institutional review board
LAZ
Length-for-age z-score
MUAC
Mid-upper arm circumference
NVP
Navirapine

J Acquir Immune Defic Syndr. Author manuscript; available in PMC 2021 October 01. 


$\begin{array}{ll}\text { PreNAPS } & \text { Prenatal Nutrition and Psychosocial Health Outcomes Study } \\ \text { PostNAPS } & \text { Postnatal Nutrition and Psychosocial Health Outcomes Study } \\ \text { WLZ } & \text { Weight-for-length z-score } \\ \text { WHO } & \text { World Health Organization }\end{array}$

\section{References}

1. Evans C, Jones CE, Prendergast AJ. HIV-exposed uninfected infants: new global challenges in the era of paediatric HIV elimination. Lancet Infect Dis. 2016;16(6):e92-e107. [PubMed: 27049574]

2. Filteau S The HIV Exposed, Uninfected African Child. Trop Med Int Heal. 2009;14(3):276-287. doi: $10.1111 / \mathrm{j} .1365$

3. Rosala-Hallas A, Bartlett JW, Filteau S. Growth of HIV-exposed uninfected, compared with HIVunexposed, Zambian children: A longitudinal analysis from infancy to school age. BMC Pediatr. 2017;17(1):1-9. doi:10.1186/s12887-017-0828-6 [PubMed: 28056921]

4. Lartey A, Marquis G, Perez-Escamilla R, Brakohiapa L, Ampofo W. Maternal HIV is Associated with Reduced Growth in the First Year of Life among Infants in the Eastern Region of Ghana: The Research to Improve Infant Nutrition and Growth (RING) Project. Matern Child Nutr. 2014;10(4):604-616. [PubMed: 22905700]

5. Sudfeld C, Lei Q, Chunyanga Y, et al. Linear growth faltering among HIV-expsoed uninfected children. J Aquir Immune Defic Syndr. 2015;25(4):368-379. doi:10.1016/ j.cogdev.2010.08.003.Personal

6. Rossouw ME, Cornell M, Cotton MF, Esser MM. Feeding practices and nutritional status of HIVexposed and HIV-unexposed infants in the Western Cape. South Afr J HIV Med. 2016;17(1):1-9. doi:10.4102/sajhivmed.v17i1.398

7. Slogrove AL. Pattern of infectious Morbidity in Hiv-exposed Uninfected infants and Children. Front Immunol. 2016;7. doi:10.3389/fimmu.2016.00164 [PubMed: 26834748]

8. Rupérez M, González R, Maculuve S, et al. Maternal HIV infection is an important health determinant in non-HIV-infected infants. Aids. 2017;31(11):1545-1553. doi:10.1097/ QAD.0000000000001499 [PubMed: 28657963]

9. Afran L, Garcia Knight M, Nduati E, Urban BC, Heyderman RS, Rowland-Jones SL. HIV-exposed uninfected children: A growing population with a vulnerable immune system? Clin Exp Immunol. 2014;176(1):11-22. doi:10.1111/cei.12251 [PubMed: 24325737]

10. Weiser SD, Young SL, Cohen CR, et al. Conceptual framework for understanding the bidirectional links between food insecurity and HIV/AIDS. Am J Clin Nutr. 2011;94(6). doi:10.3945/ ajcn.111.012070

11. Ajo K, Ojofeitimi E, Adebayo A, Fatusi A, Afolabi O. Influence of family size, household food security status, and child care practices on the nutritional status of under-five. Afrcan J Feproductive Heal. 2010;14(4):123-132.

12. Saha KK, Frongillo EA, Alam DS, Arifeen SE, Rasmussen KM, Lars A. Household food security is associated with growth of infants and young children in rural Bangladesh. Public Health Nutr. 2009;12(9):1556-1562. doi:10.1017/S1368980009004765 [PubMed: 19232147]

13. Widen EM, Collins SM, Khan H, et al. Food insecurity, but not HIV-infection status, is associated with adverse changes in body composition during lactation in Ugandan women of mixed HIV status 1 - 3. Am J Clin Nutr. 2017;105:361-368. doi:10.3945/ajcn.116.142513.1 [PubMed: 28052888]

14. Webb-Girard A, Cherobon A, Mbugua S, Kamau-Mbuthia E, Amin A, Sellen DW. Food insecurity is associated with attitudes towards exclusive breastfeeding among women in urban Kenya. Matern Child Nutr. 2012;8(2):199-214. doi:10.1111/j.1740-8709.2010.00272.x [PubMed: 20874844]

15. Miller JD, Young SL, Boateng GO, Oiye S, Owino V. Greater household food insecurity is associated with lower breast milk intake among infants in western Kenya. Matern Child Nutr. 2019;15(4):1-8. doi:10.1111/mcn.12862 
16. World Health Organization. Use of antiretroviral drugs for treating pregnant women and preventing HIV infection in infants. Geneva WHO. 2012;(4):1-117. doi:WHO/HIV/2012.6

17. World Health Organization. Guideline: Updates on HIV and infant feeding. 2016.

18. Jao J, Abrams EJ. Metabolic complications of in utero maternal HIV and antiretroviral exposure in HIV-exposed infants. Pediatr Infect Dis J. 2014;33(7):734-740. doi:10.1097/ INF.0000000000000224 [PubMed: 24378947]

19. Desmonde S, Goetghebuer T, Thorne C, Leroy V. Health and survival of HIV perinatally exposed but uninfected children born to HIV-infected mothers. Curr Opin HIV AIDS. 2016;11(5):465-476. doi:10.1097/COH.0000000000000300 [PubMed: 27716731]

20. Denneman L, Cohen S, Godfried M, et al. Exposure to improved nutrition from conception to age two years and adult cardiometabolic disease risk. Lancet Glob Heal. 2018;in press(8):e875-e884. doi:10.1016/S2214-109X(18)30231-6

21. Powis K, Seaton L, Ogqu A, Lockman S, Dryden-Peterson S, van Widenfelt E. Effects of in Utero Antiretroviral Exposure on Longitudinal Growth of HIV-exposed Uninfected Infants in Botswana. J Acquir Immune Defic Syndr. 2011;56(2):131-138. [PubMed: 21124227]

22. White M, Feucht UD, Duffley E, et al. Does in utero HIV-exposure influence infant development and immune outcomes? Findings from a piolt study in Pretoria, South Africa. medRxiv. 2019;19003889:1-16. doi:10.1017/CBO9781107415324.004

23. Brennan AT, Bonawitz R, Gill CJ, et al. A meta-analysis assessing all-cause mortality in HIVexposed uninfected compared with HIV-unexposed uninfected infants and children. AIDS. 2016;30(15):2351-2360. doi:10.1097/QAD.0000000000001211 [PubMed: 27456985]

24. Pierre R, Fulford T, Lewis K, Palmer P, Walters C, C C. Infectious disease morbidity and growth among young HIV-exposed and uninfected children in Jamaca. Rev Panam Salud Publica. 2016;40(6):401-409. [PubMed: 28718488]

25. Goldberg RE, Short SE. What do we know about children living with HIV- infected or AIDS-ill adults in Sub-Saharan Africa? A systematic review of the literature What do we know about children living with HIV-infected or AIDS-ill adults in Sub-. AIDS Care. 2016;28(supp 2):130141. doi:10.1080/09540121.2016.1176684 [PubMed: 27392008]

26. Jao J, Powis KM, Kirmse B, et al. Lower mitochondrial DNA and altered mitochondrial fuel metabolism in HIV-exposed uninfected infants in Cameroon. Aids. 2017;31(18):2475-2481. doi:10.1097/QAD.0000000000001647 [PubMed: 28926411]

27. Jao J, Kirmse B, Yu C, et al. Lower preprandial insulin and altered fuel use in HIV/antiretroviralexposed infants in Cameroon. J Clin Endocrinol Metab. 2015;100(9):3260-3269. doi:10.1210/ JC.2015-2198 [PubMed: 26133363]

28. Bender JM, Li F, Martelly S, et al. Maternal HIV infection influences the microbiome of HIVuninfected infants. Sci Transl Med. 2016;8(349). doi:10.1126/scitranslmed.aaf5103

29. Lane CE, Bobrow EA, Ndatimana D, Ndayisaba GF, Adair LS. Determinants of growth in HIVexposed and HIV-uninfected infants in the Kabeho Study. Matern Child Nutr. 2019;15(3). doi: $10.1111 / \mathrm{mcn} .12776$

30. Jumare J, Datong P, Osawe S, et al. Compromised Growth among HIV Exposed Uninfected Compared to Unexposed Children in Nigeria. Pediatr Infect Dis J. 2019;38(3):1. doi:10.1097/ inf.0000000000002238 [PubMed: 30204658]

31. le Roux SM, Abrams EJ, Donald KA, et al. Growth trajectories of breastfed HIV-exposed uninfected and HIV-unexposed children under conditions of universal maternal antiretroviral therapy: a prospective study. Lancet Child Adolesc Heal. 2019;3(4).

32. Ram N, Grimm KJ. Growth mixture modeling: A method for identifying differences in longitudinal change among unobserved groups. Int J Behav Dev. 2009;33(6):565-576. doi:10.1177/0165025409343765 [PubMed: 23885133]

33. Jung T, Wickrama KAS. An Introduction to Latent Class Growth Analysis and Growth Mixture Modeling. Soc Personal Psychol Compass. 2008;2(1):302-317. doi:10.1111/ j.1751-9004.2007.00054.x

34. Natamba B, Achan J, Stoltzfus R, Griffiths J, Young S. The association between food insecurity and depressive symptoms severity among pregnant women differs by social support category: a cross-sectional study. Matern Child Nutr. 2016;8 9. 
35. Natamba BK, Achan J, Arbach A, et al. Reliability and validity of the center for epidemiologic studies-depression scale in screening for depression among HIV-infected and -uninfected pregnant women attending antenatal services in northern Uganda : a cross-sectional study. BMC Psychiatry. 2014; 14 .

36. Natamba B, Kilama H, Arbach A, Achan J, Griffiths J, Young S. Reliability and validity of an invidually focused food insecurity access scale for assessing inadequate access to food among pregnant Ugandan women of mixed HIV status. Public Health Nutr. 2015;18:2895-2905. [PubMed: 25171462]

37. Familiar I, Collins S, Sikorskii A, et al. Quality of caregiving is positively associated with neurodevelopment during the first year of lie among HIV-exposed uninfected children in Uganda. Physiol Behav. 2017;176(3):139-148. doi:10.1016/j.physbeh.2017.03.040 [PubMed: 28363838]

38. World Health Organization. WHO Anthro: igrowup. 2011.

39. Prost-Lima C, Phillips V, Diakite A, Liquet B. Lcmm: Extended mixed models using latent classes and latent processes. 2017.

40. Wickham H ggplot2: elegant graphics for data analysis. 2016.

41. Crossant Y Mlogit: Multiple Logit mModel. 2013.

42. Bates D, Machler M, Bolker B, Walker S. Fitting linear mixed-effects models using lme4. J Stat Softw. 2015;67.

43. Dewey KG, Begum K. Long-term consequences of stunting in early life. Matern Child Nutr. 2011;7(Suppl. 3):5-18. doi:10.1111/j.1740-8709.2011.00349.x [PubMed: 21929633]

44. Crookston BT, Penny ME, Alder SC, et al. Children Who Recover from Early Stunting and Children Who Are Not Stunted Demonstrate Similar Levels of Cognition. J Nutr. 2010;140(11):1996-2001. doi:10.3945/jn.109.118927 [PubMed: 20844188]

45. WHO WOrking Group. Use and interpretation of anthropometric indicators of nutritional status. Bull World Health Organ. 1986;64(6):929-941. [PubMed: 3493862]

46. National Institute of statistics, Ministry of Health, ICF International, National Institute of statistics, $\mathrm{M}$ of H [Rwanda], ICF International Rwanda Demographic and Health Survey. Rockville, Maryland, USA: NISR, MOH, and ICF International; 2015. doi:3, 2016

47. Uganda Bureau of Statistics, ICF. Uganda Demographic and Health Survey 2016. Kampala, Uganda and Rockville, Maryland, Uganda and Rockville, Maryland: UBOS and ICF; 2018. 

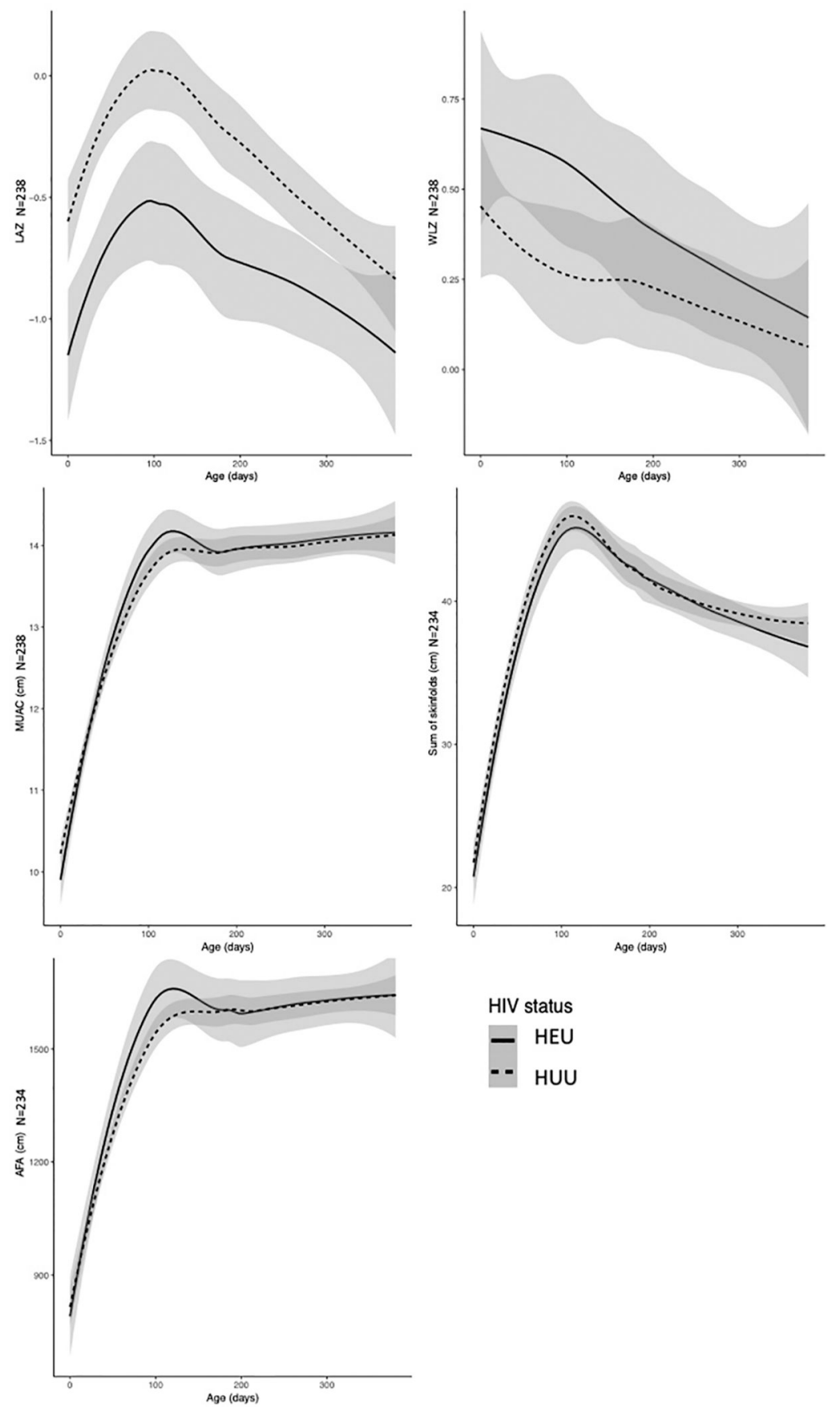

HIV status
- HEU
-

Figure 1:

HEU infants have lower mean LAZ through the first year of life. WLZ, MUAC, sum of skinfolds, and AFA are similar between HEU and HUU infants. 

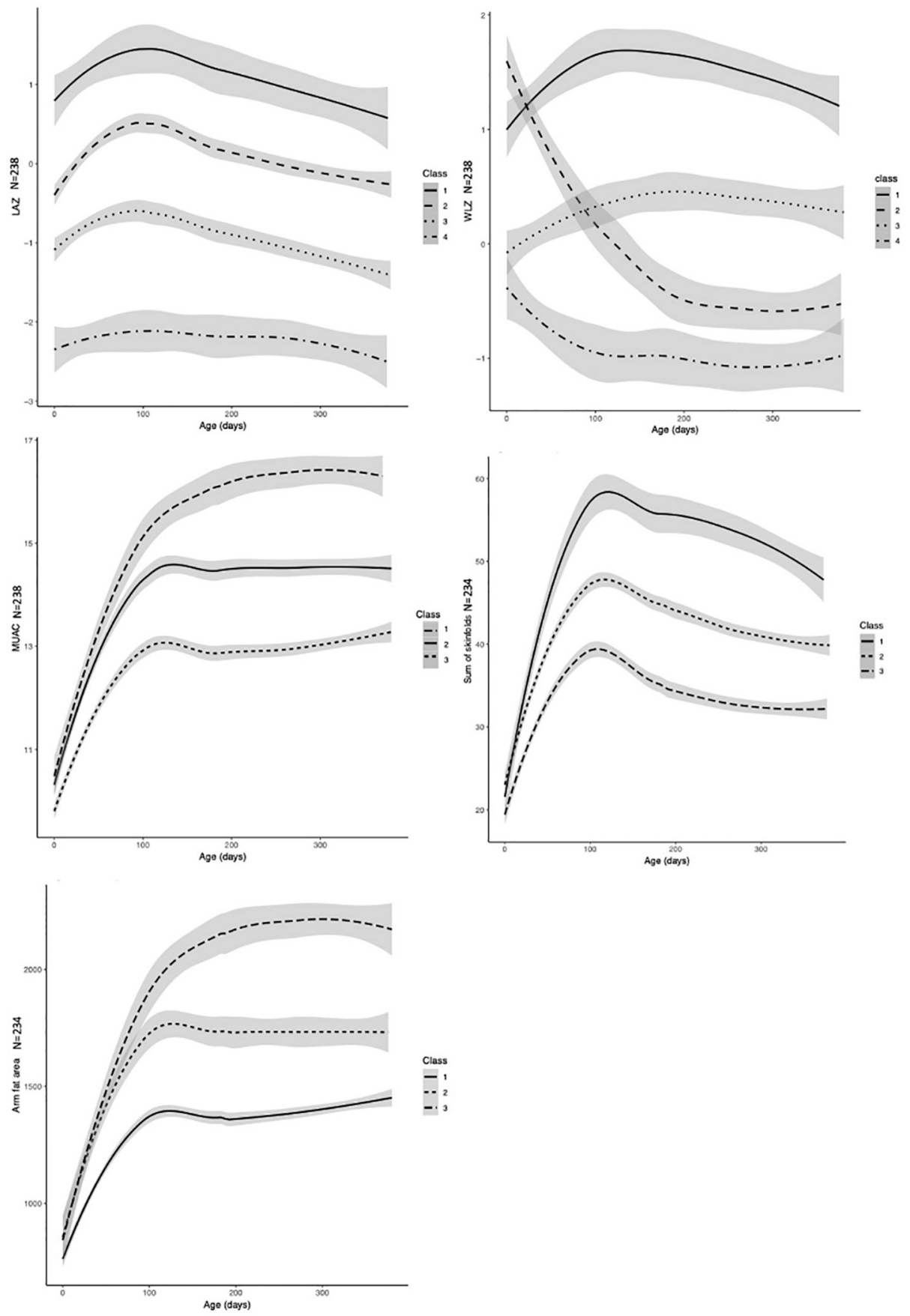

Figure 2:

Mean growth trends of classes by anthropometric measure indicate that some subgroups experience fundamental differences in the shapes of their growth over time. 
Table 1:

Characteristics of mother-infant dyads in PostNAPS included in analytic sample, by maternal HIV status $(n=238)$. Presented as percent and mean. Standard deviations are included in parentheses for continuous values.

\begin{tabular}{|c|c|c|c|}
\hline & HIV + mother & HIV - mother & P-value comparing $+/-$ mothers \\
\hline $\mathrm{N}$ & $36 \%^{1}$ & $63 \%$ & \\
\hline \multicolumn{4}{|l|}{ Infant } \\
\hline Female sex & $43 \%$ & $47 \%$ & 0.59 \\
\hline Born in hungry season & $30 \%$ & $38 \%$ & 0.26 \\
\hline Gestational age & $39.14(2.4)$ & $39.44(2.3)$ & 0.36 \\
\hline \multicolumn{4}{|l|}{ Breastfeeding } \\
\hline EBF at baseline & $83 \%$ & $72 \%$ & 0.02 \\
\hline $\mathrm{EBF}$ at $3 \mathrm{mo}$ & $59 \%$ & $42 \%$ & 0.03 \\
\hline $\mathrm{EBF}$ at $6 \mathrm{mo}$ & $17 \%$ & $8 \%$ & 0.08 \\
\hline \multicolumn{4}{|l|}{ Anthropometrics } \\
\hline Birthweight, kg & $3.4(5.0)$ & $3.4(4.8)$ & 0.63 \\
\hline LAZ at 1 month & $-0.7(1.3)$ & $-0.1(1.1)$ & $<0.001$ \\
\hline LAZ at 9.5 months & $-0.8(1.1)$ & $-0.5(1.1)$ & 0.05 \\
\hline WLZ at 1 month & $0.6(1.3)$ & $0.3(1.3)$ & 0.15 \\
\hline WLZ at 9.5 months & $0.3(1.2)$ & $0.1(1.2)$ & 0.24 \\
\hline MUAC at 1 month & $11.8(1.0)$ & $11.9(1.0)$ & 0.26 \\
\hline MUAC at 9.5 months & $14.1(1.2)$ & $14.0(1.3)$ & 0.68 \\
\hline Sum of skinfolds at 1 month & $29.2(8.1)$ & $30.4(7.5)$ & 0.02 \\
\hline Sum of skinfolds at 9.5 months & $38.0(8.6)$ & $38.9(7.6)$ & 0.99 \\
\hline AFA at 1 month & $1444.8(215.6)$ & $1437.0(195.6)$ & 0.80 \\
\hline AFA at 9.5 months & $1640.8(285.7)$ & $1627.5(307.6)$ & 0.47 \\
\hline \multicolumn{4}{|l|}{ Maternal } \\
\hline Age, y & $25.8(5.6)$ & $24.9(5.2)$ & 0.19 \\
\hline Education beyond primary school & $38 \%$ & $51 \%$ & 0.06 \\
\hline Height, cm & $162.6(5.8)$ & $162.6(6.2)$ & 0.97 \\
\hline BMI, $\mathrm{kg} / \mathrm{m} 2$ at first prenatal visit & $22.9(3.1)$ & $22.9(2.9)$ & 0.88 \\
\hline Asset score & $-0.3(1.4)$ & $0.2(1.6)$ & 0.02 \\
\hline Food insecurity score & $6.8(5.4)$ & $5.2(4.1)$ & 0.01 \\
\hline Was displaced & $55 \%$ & $53 \%$ & 0.77 \\
\hline Housewife & $50 \%$ & $45 \%$ & 0.44 \\
\hline
\end{tabular}

${ }^{1 .}$ This percentage reflects the percent of the whole whereas the other percentages are calculated as the percent within each exposure category.

2. Bold reflects p-value $<0.05$ 


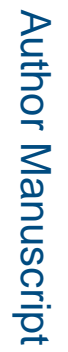

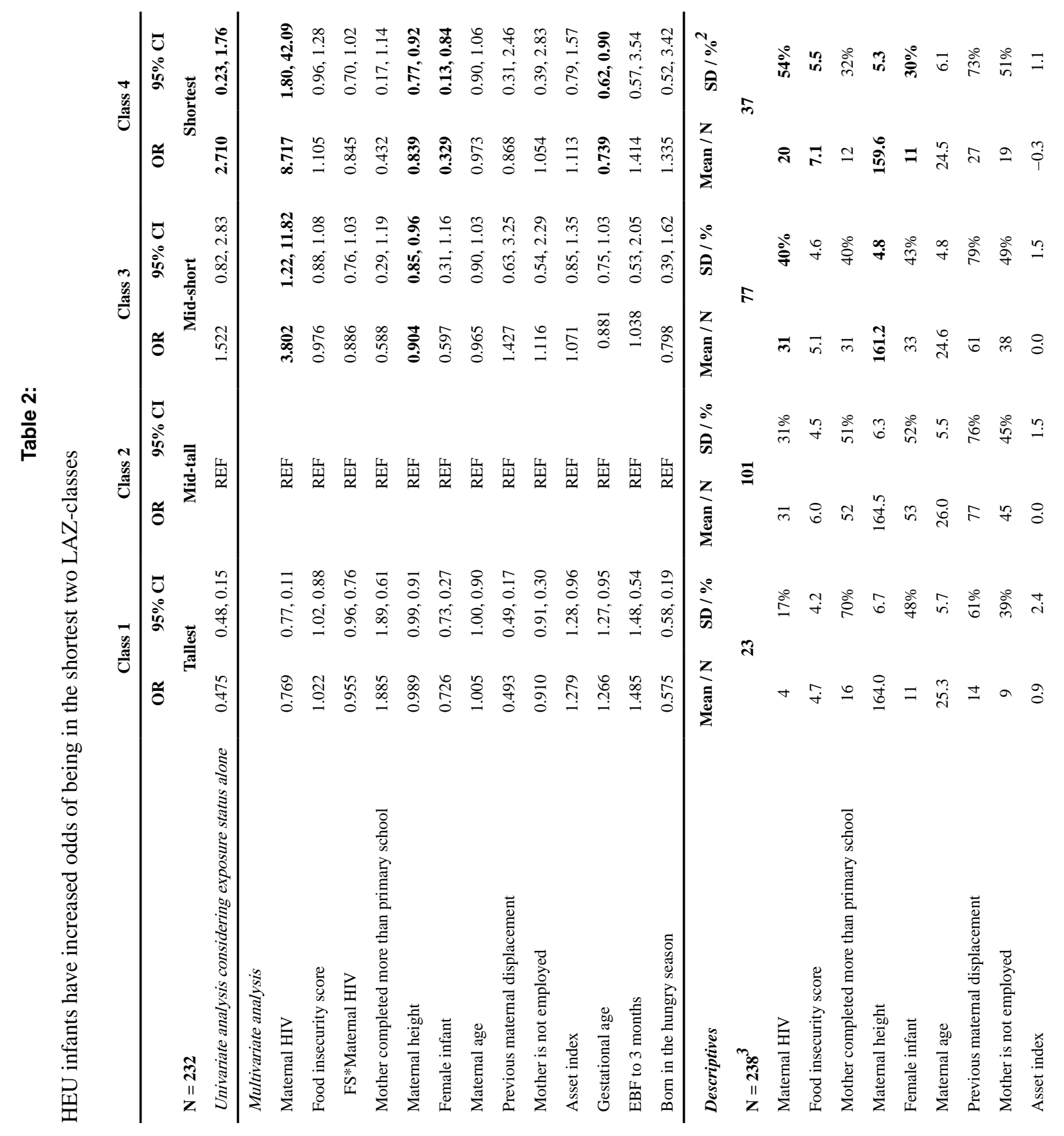

J Acquir Immune Defic Syndr. Author manuscript; available in PMC 2021 October 01. 


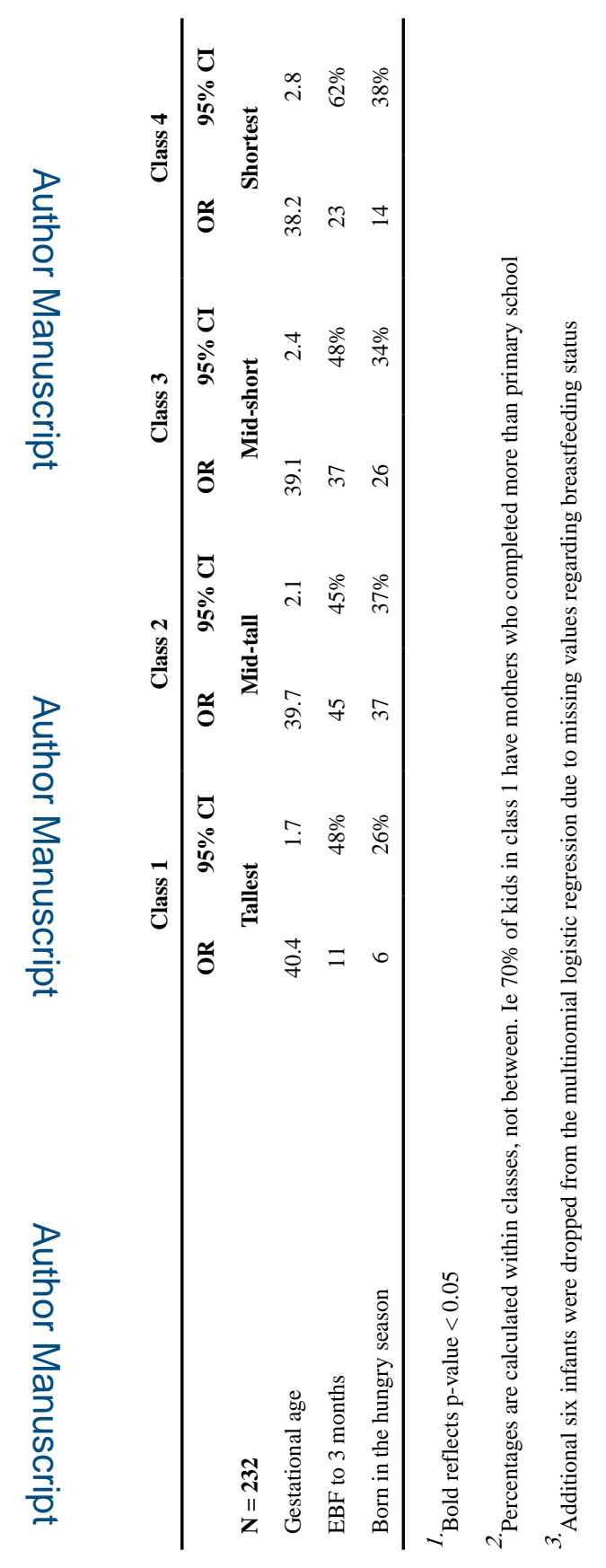

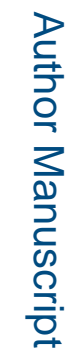




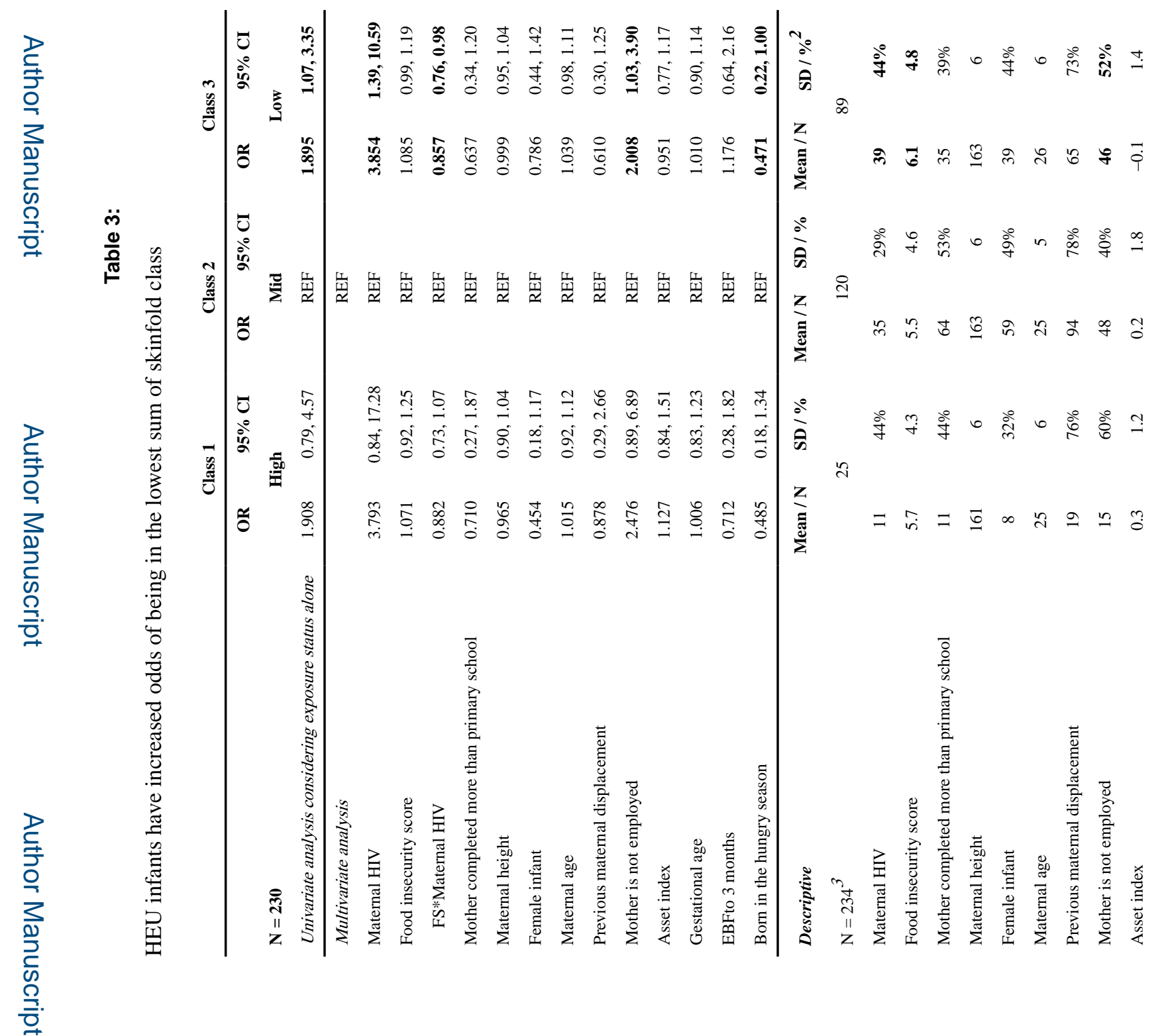




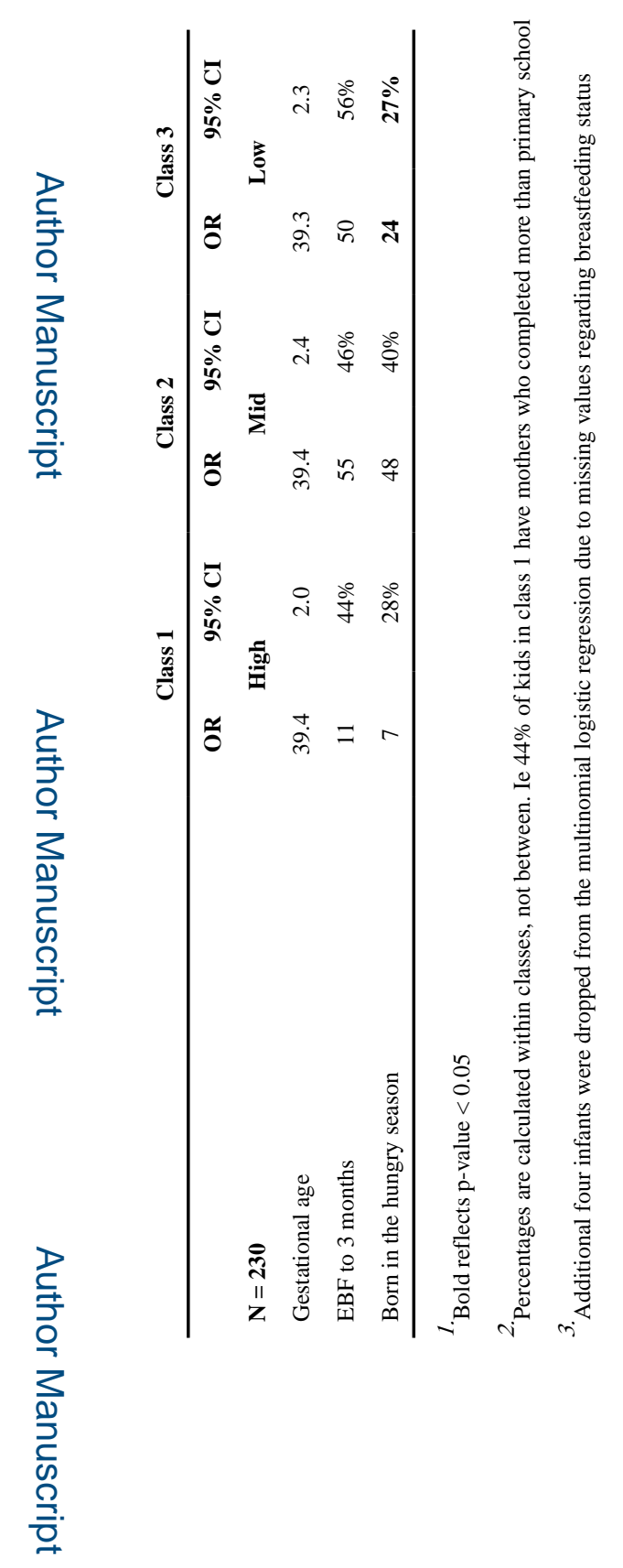

로을 